\title{
PREFACE
}

\section{PHILOSOPHIE DE LA SINGULARITÉ}

\author{
RENÉ THOM \\ Institut des Hautes Études Scientifiques, \\ Bures-sur-Yvette, France
}

Quand S. Lojasiewicz m'a proposé d'écrire une préface à ce volume consacré aux résultats les plus récents en théorie des singularités - j'ai eu d'abord quelque réticences. Il m'a alors demandé d'expliciter en quoi et pourquoi la théorie des singulurités me semblait importante - fût-ce sur un plan qui, débordant la mathématique, irait jusqu'à la philosophie; je n'ai pu que céder, dès lors, à son aimable insistance. Je me propose donc d'expliquer ici rapidement - in a nutshell - cornment et pourquoi la singularité mathématiquement définie intervient dans notre description et notre compréhension du monde réel.

\section{Mathématique et prédiction}

Tout un chacun admet, que du point de vue pragmatique, une tâche essentielle de la science est la prédiction des phénomènes. Or prédire, c'est extraire d'une connaissance du passé une connaissance de l'avenir. C'est donc, mathématiquement parlant, l'extrapolation d'une fonction $f(t)$ connue pour $t<0$ (sur le passé noté $t^{-}$) au futur $t>0$ (noté $t^{+}$). Or parmi les procédés canoniques d'extrapolation qu'offre la mathématique, il n'en est guère qu'un qui s'impose par son caractère canonique, c'est le prolongement analytique. En effet, il n'est pas raisonnable de penser que l'instant présent $(t=0)$ joue un rôle exceptionnel, ce qui revient à dire que l'opérateur qui fait passer de $f\left(t^{-}\right)$à $f\left(t^{+}\right)$ne peut être de nature arbitraire, car pour $t^{-}, t^{+}$ tendant vers zéro, cet opérateur tend vers l'identité; il en résulte que si $f$ est analytique, cet opérateur ne peut être que lidentité. 
Si l'on accepte ce point de vue, supposant que l'étude du passé ait pu nous fournir la fonction $f\left(t^{-}\right)$en tant que fonction analytique, alors le prolongement analytique de $f$ permettra la prédiction sur $t^{+}$. Ceci tout au moins tant que ce prolongement reste possible. Bien entendu, le domaine d'holomorphie va présenter des singularités qui arrêtent le prolongement aux frontières du domaine. De ce point de vue, les premières singularités sont celles qui limitent le domaine d'holomorphie. Certaines de ces singularités sont bénignes: par exemple le pôle $z=0$ d'une fonction méromorphe comme $1 / z$. On peut contourner une telle singularité. Parfois en contournant une singularité telle que $\sqrt{\xi}$, on introduira une multiformité de la fonction, ce qui peut conduire à une indétermination pratique de la solution.

Observons enfin que dans $C$, le théorème d'uniformisation de Riemann nous apprend que tout disque ouvert est un domaine d'holomorphie. En ce sens, l'ensemble frontière d'un domaine d'holomorphie dans $C$ peut être topologiquement très arbitraire, et il n'est pas raisonnable d'espérer tirer une classification complète des "accidents" possibles qu'il peut présenter. Mais dans $C^{2}, \ldots, C r, n>2$, on sait que la frontière du domaine d'holomorphie doit satisfaire à des conditions de $q$-convexité qui, si cet ensemble est assez lisse, sont pratiquement suffisantes (théorème d'Oka).

\section{Pourquoi l'analyticité?}

Ce point de vue pourrait conduire à l'objection suivante: si vraiment toute prédiction en Science se ramène - en dernière analyse - à l'usage du prolongement analytique, alors à quoi servent physiciens, chimistes, biologistes, en un mot à quoi sert la science expérimentale? Leur tâche se réduit-elle à fournir la base numérique de départ $f\left(t^{-}\right)$qu'on va extrapoler? Ne serait-ce pas là donner aux mathématiciens un trop beau rôle? De plus, pourquoi penser que toute prédiction se fonde sur l'analyticité? N'y a-t-il pas des opérateurs qui fonctionnent par exemple en catégorie $(\zeta)^{x}$, ou $(/)^{2}$, comme dans la théorie des opérateurs hyperboliques? Pourquoi notre univers seraitil modélisable par un être analytique?

On pourrait répondre - non sans raison - que l'emploi en Physique de lanalyticité ressort souvent de ce qu'on a appelé la "Philosophie du lampadaire"(1). Mais il y a, je crois, des réponses un peu plus satisfaisantes à donner. En ce qui concerne les extrapolations non analytiques, les opérateurs agissant en classe $"{ }^{\infty}$ ou classe ${ }^{\prime}{ }^{2}$ sont en général des extensions naturelles d'opérateurs "analytiques", qui transforment les fonctions analytiques en fonction analytiques. Dans le cas du théorème de préparation $4^{\infty}$, on a une

(1) Allusion à l'anecdote classique du monsieur qui a perdu son portemonnaie dans une rue obscure, et va le rechercher sous la clarté du lampadaire, alors qu'il sait fort bien l'avoir perdu ailleurs. 
extension non canonique (et indéterminée) de l'opérateur analytique. En Physique Fondamentale, l'usage de l'analyticité est motivé par le caractère analytique des représentations des groupes de symétrie (externes ou internes) qui interviennent en théorie des particules et de leurs interactions. Or ce caractère analytique est prouvé dans le cas où le groupe de symétrie $G$ considéré est compact (théorème de Peter-Weyl). Bien entendu, le groupe à un paramètre du temps $\boldsymbol{R}$ est non compact, et toute la richesse de la dynamique qualitative vient de là. Mais en Physique, et en d'autres sciences également, il est d'usage de s'intéresser aux "régimes asymptotiques", en oubliant les régimes transitoires qui y aboutissent. En ce cas, on exorcise le caractère non compact de $\boldsymbol{R}$ du groupe des translations par une représentation appropriée dans un groupe compact. Ainsi la motivation profonde de la Mécanique Quantique réside dans le théorème de von Neumann qui dit qu'une représentation de l'algèbre de Lie commutative $(q, p)$ dans le groupe projectif complexe $\operatorname{Proj}(k)$ se relève en une représentation du groupe de Heisenberg dans le groupe unitaire de même dimension $k$.

Mais même en acceptant le postulat d'analyticité universelle, on n'a pas résolu tous les problèmes; en effet une donnée expérimentale $f\left(t^{-}\right)$est toujours par définition approchée. Or on sait très bien que dans tout voisinage d'une fonction $f$ sur un compact en topologie $\mathscr{C}^{\infty}$ ou $\mathscr{C}^{k}$, il y a des fonctions analytiques $g$ dont le domaine d'holomorphie est très différent de celui de $(f)$. Cette instabilité de la frontière du domaine d'holomorphie ruine en fait toute possibilité d'une prédiction précise fondée sur des données uniquement expérimentales. Si la Physique réussit, c'est parce qu'elle fait des hypothèses ontologiques sur la nature ultime du réel, d'ampleur tout à fait cosmique. Seule une théorie permet de restreindre "a priori" la classe des fonctions $f\left(t^{-}\right)$de telle manière que cette instabilité des domaines d'holomorphie puisse être efficacement contrôlée, et qu'ainsi puisse s'obtenir une bonne précision des prédictions.

\section{Description et classification}

On verra d’abord dans la singularité une notion purement statique. On prospecte un espace dont on jauge l'homogénéité ou - au contraire - dont on examine les hétérogénéités. On range dans une même classe d'équivalence deux points $x, y$ pourvus de voisinages $U_{x}, U_{y}$ qui du point de vue de leur propriétés locales peuvent être déformés continûment l'un dans l'autre sans qu'à aucun moment il n'y ait rupture dans le type local (algébrique ou topologique) de ces voisinages locaux. On est ainsi amené naturellement à la notion de stratification. Dans les "bons" cas (ensembles analytiques, semianalytiques, sous-analytiques), les classes d'équivalence ainsi définies sont des 
sous-variétés plongées, les "strates", dont les incidences relatives sont astreintes à certaines conditions de continuité tangentielle. Ce fut l'immense mérite de Hassler Whitney que de spécifier les critères différentiels (propriétés a), b)) qui, dans les bons cas, permettent d'établir l'existence de stratifications douées de bonnes propriétés: par exemple, on peut définir ce qu'est une application $f$ d'une variété sur un ensemble stratifié $K \subset \boldsymbol{R}^{n}$ : les contre-images $f^{-1}(K)$ sont alors des ensembles stratifiés présentant la stabilité topologique pour presque toute $f$. Un morphisme analytique propre $\varphi: A \rightarrow B$ peut être stratifié dans le sens faible qu'il existe des stratifications $A=\bigcup_{i} X_{i}, B=\bigcup_{j} Y_{j}$ telles que $f \mid X_{i}$ soit une surjection sur une strate image $Y_{j(i)}$. Mais seuls les morphismes "sans éclatement" ont de bonnes propriétés topologiques, en ce sens que des déformations $\varphi_{\lambda}$ de $\varphi, \lambda \in I$ qui définissent des stratifications produits de $A \times I \rightarrow B \times I$ conduisent à l'invariance du type de $\varphi_{\lambda}$. Dans le cas général où il $y$ a de l'éclatement (i.e., où certaines strates de $A$ subissent un "collapse" supérieur à celui de strates de leur étoile), ceci n'est plus vrai en général, et une notion fine de morphisme stratifié, en ce cas, reste à définir. Si le formalisme des ensembles stratifiés (plongés dans $\boldsymbol{R}^{n}$, donc séparés) est maintenant bien compris, par contre on peut rêver d'une théorie des ensembles stratifiés non séparés, qui apparemment interviennent dans la description de quotients par des groupes non compacts fort simples. Par exemple, le quotient de $\boldsymbol{R}^{n}$ par un automorphisme linéaire hyperbolique. La théorie des stratifications de ce point de vue, n'a sans doute pas dit son dernier mot.

\section{Singularités et processus propagatifs}

Nous venons d'explorer la singularité comme une forme statique, permanente. Ceci n'est qu'un aspect de la notion de singularité. En fait, la singularité est toujours liée, de manière explicite ou implicite, à un processus global, de nature propagative défini sur l'espace ambiant au voisinage de la forme. La singularité apparait donc comme obstacle (ou liée à un obstacle) s'opposant à la propagation d'un processus spatial. Comme les entités du monde extérieur sont - très souvent - détectées comme des obstacles à notre action, on comprend pourquoi la notion de singularité apparait toutes les fois qu'on veut prouver en Science la présence d'une entité extérieure à nous. Les modèles théoriques qui seuls, selon le partie 2, permettent de stabiliser le prolongement analytique reposent toujours sur la présence postulée d'entités théoriques (particules, champs ...) dont la manifestation empirique est l'obstacle à l'action (qu'on songe par exemple aux effets de diffusion - scattering - dans les expériences de collision). Mais deux cas, assez différents, sont à considérer. 
4.1. La singularité comme engendrée par le confit d'un fux avec une forme-obstacle. On considère une forme spatiale $(F)$ immergée dans et impénétrable à un flux (de matière ou d'énergie) parcourant l'espace au voisinage de $(F)$. Ce flux vient heurter la forme $(F)$ et tend à l'entraîner. Mais la forme $(F)$ est tixée (en principe) et refuse de se laisser emporter; elle va créer dès lors dans le flux des modifications de direction, de densité, et d'amplitude dont certaines pourront persister indéfiniment en aval de l'obstacle. Ce schéma rend compte d'un processus indispensable à l'expérience: l'observation. Comme nous l'apprend la Physique, l'observation implique toujours un observateur, et toute observation visuelle est en dernière analyse une projection géométrique $(p)$ (par exemple, sur cette surface sphérique qu'est la rétine de l'observateur). (En Mécanique Quantique, également, tout mesurement est un projecteur $p^{2}=p$ ). De là vient que nous ne voyons jamais les choses elles-mêmes, mais seulement leurs contours apparents, i.e. l'ensemble des valeurs critiques de la projection $p$ restreinte à la forme $(F)$. Telle est en particulier la situation de la théorie de Morse, où une fonction réelle lisse applique une variété $M$ dans l'axe réel $\boldsymbol{R}$. La variété $M$ résiste à cet aplatissement, et manifeste son opposition au flux $p$ en cristallisant des points critiques, dont la disposition relative et l'indice permettent de reconstituer la topologie de $M$. Les contours apparents présentent en général des singularités, dont certaines sont "génériques", c'està-dire qu'elles résistent à des déformations de la position de $(F)$ ou de la projection $p$. Pour chaque couple $(n, k)$, il n'y a pour les applications lisses de $\left(R^{n}, 0\right)$ dans $\left(R^{k}, 0_{1}\right)$ qu'un ensemble fini de types (topologiques) de singularités génériques. Les travaux récents (J. Mather, Arnol'd et son école) ont beaucoup fait pour préciser cette phénoménologie des singularités. On observera, à cet égard, qu'en Analyse Fonctionnelle, le spectre d'un opérateur n'est jamais qu'un ensemble de valeurs critiques, un "contour apparent".

Comme les prisonniers de la caverne de Platon, nous ne voyons jamais que les ombres des choses (leurs contours apparents). Il importe, connaissant les contours apparents d'une forme $(F)$, de savoir reconstituer cette forme. On interprétera de cette manière les théorèmes de désingularisation de Hironaka. Etant donné uñ "bon" ensemble $E$ plongé dans un espace euclidien $\boldsymbol{R}^{k}$, on le considérera comme la projection d'un ensemble plus simple $\tilde{E}$ plongé dans $\boldsymbol{R}^{n}$ pour une projection lineaire $p=\boldsymbol{R}^{\boldsymbol{n}} \rightarrow \boldsymbol{R}^{k}$. On cherche à rendre $\tilde{E}$ aussi proche que possible d'une variété lisse. Le théorème de Hironaka montre qu'on ne peut éviter les croisements normaux (sur $C$ ) ou les variétés à coins (sur $R$ ). Or pour un ensemble stratifié $K$ plongé dans un espace euclidien $\boldsymbol{R}^{m}$, on peut remplacer chaque strate de $K$ par son voisinage . tubulaire, et tous ces tubes se coupent transversalement. L'ensemble $K$ peut ainsi être défini comme une concaténation (selon un schéma d'incidence) de variétés à coins. Cette persistance des coins et des croisements normaux est l'un des faits les plus mystérieux des singularités analytiques. 
Comme un phénomène varie dans son apparence lorsque varie la position de l'observateur, tout phénomène peut être regardé comme une classe d'équivalence entre apparences locales. Or la réalisation géométrique standard d'une relation d'équivalence dans un espace euclidien est le feuilletage. D'où l'importance des singularités de feuilletages, des projections de feuilletages, ou des structures bifeuilletées. De même pour les singularités des formes differentielles et des champs de vecteurs. Ces théories, qui ne vont guère au-delà de la situation générique, sont encore dans une prime enfance, et réservent un beau matériel aux mathématiciens de l'avenir.

4.2. Dans un second cas, la singularité apparaît comme due à un conflit de la structure propagative ambiante avec elle-même; autrement dit, la singularité est engendrée par le processus propagatif qu'elle limite. Tel est évidemment le cas des singularités-frontières d'un domaine d'holomorphie. On citera aussi le cas des points singuliers d'un flot sur une variété, dont on sait qu’ils existent nécessairement si la variété a une caractéristique d'Euler non nulle. De manière générale, lorsqu'on veut construire une section d'un fibré, on peut toujours le faire à l'exception d'un ensemble obstruction de la base. On s'efforce alors de déterminer la forme générique, structurellement stable de cette obstruction; les singularités ont alors un "indice" non nul, défini comme une classe d'homotopie non nulle du link de l'ensemble singulier dans la variété des structures locales régulières. Ces constructions mathématiques ont des équivalents dans le monde physique: les caustiques de l'Optique géométrique (alias variétés lagrangiennes), les ondes de choc des écoulements supersoniques. Enfin les structures ordonnées de la matière (cristaux, nématiques, smectiques) présentent en général des interruptions qu'on appelle des "défauts". Une theorie des défauts stables existe, qui est l'analogue de la théorie de l'indice pour les singularités des sections de fibrés (règle de Kléman-Toulouse en France, de Volovik-Mineev en URSS).

\section{La singularité comme générateur local de formes}

On aurait tort de voir dans la singularité le seul effet de l'incapacité d'un milieu spatial à accepter une certaine structure globale. On peut avoir le point de vue inverse, et prétendre qu'une singularité a un pouvoir génératif qui lui permet de structurer l'espace environnant. C'est, en tout cas, ce qui se passe en Algèbre. Un polynome $P(x)$ est déterminé par l'ensemble de ses zéros (sur $C$ ). Une fonction méromorphe l'est, à un facteur inversible près, par. l'ensemble de ses pôles et de ses zéros. Un ensemble algébrique $A$ peut être vu comme un fermé statique de l'espace mais il peut aussi engendrer un idéal $\mathscr{J}$ de fonctions partout définies. Du point de vue de l'algèbre (au moins sur les complexes), il y a une quasi-parfaite équivalence entre le point de vue ensembliste et le point de vue des algèbres locales de fonctions. 
Mais il est encore un autre point de vue qui attribue à la singularité un pouvoir structurant sur son environnement; les ensembles singuliers des contours apparents ont une structure stratifiée qui manifeste une hiérarchie de singularités. On peut considérer la fibre au-dessus d'un point générique $v$, soit $F_{v}$, comme une déformation (en général plate) de la fibre singulière centrale $F_{0}=p^{-1}(0)$. De là lintroduction de la théorie de la déformation universelle d'un germe d'hypersurface analytique, l'espace du déploiement universel est lisse, et de dimension finie. D'où la possibilité d'utiliser une nouvelle forme - strictement ponctuelle - de prolongement analytique, où on étend l'espace des variables initiales (variables "internes") par les variables "externes" qui paramétrisent le déploiement. C'est là le principe de la théorie des catastrophes élémentaires, dont on sait qu'elle a quelques applications convaincantes pour décrire (sinon prédire) des phénomènes tels que les conflits de régimes, et les singularités des caustiques (projections des variétés lagrangiennes).

En physique, une particule chargée est source d'un champ: on peut dire que la particule génère son champ par une sorte d'éclatement permanent. De même, en théorie des structures ordonnées, certains défauts sont des lieux privilégiés par où s'accroît ou se désagrège la structure; ainsi, une dislocation - vis d'un cristal peut être à l'origine d'un "whisker", zone de croissance rapide due à laccrétion du cristal le long de l'axe de la dislocation.

Pour terminer cette énumération - nullement exhaustive - des emplois des singularités, je voudrais citer le rôle joué par les singularités comme "indices" porteurs de signification dans le monde biologique et humain. Ce qui compte, ici, c'est de remonter à l'origine du "codage": il s'agit toujours, en dernière analyse, de l'effet d'arrêt, de naissance, de cassure dichotomique ou de confluence exercé par un obstacle dans l'écoulement d'un flux. (Par exemple: ouvrir une porte assure une connexion entre les deux chambres qu'elle sépare). Ces singularités "archétypes" jouent un rôle considérable (rarement perçu parce qu'implicite) dans l'organogenèse en Embryologie, ou dans la conception, et la fabrication de nos outils. Il en va de même pour les effets causatifs (excitation, inhibition) décrits dans les schémas cybernétiques. Nous entrons là dans un domaine mal connu, où les mathématiciens soucieux de trouver des correspondances dynamiques aux schémas logiques ou booléens ont encore peu pénétré.

Conclusion. Cette opposition entre une singularité créée comme un défaut d'une structure propagative ambiante, ou une singularité qui est source de l'effet propagatif lui-même pose un problème central qu'on retrouve pratiquement à l'intérieur de presque toutes les disciplines scientifiques. La Physique contemporaine admet plutôt le premier aspect: la particule est source d'un champ qu'elle génère; Einstein, en Relativité 
Générale, verra plutôt dans la particule la singularité d'une métrique de l'espace-temps. On retrouve ici cette aporie fondamentale du continu et discret qui est au coeur de la mathématique. On retrouvera cette même aporie jusqu'en psychologie: est-ce que nous parlons parce que nous pensons, ou au contraire est-ce que nous pensons parce que nous parlons? 